\title{
LA HIBRIDACIÓN DE MODELOS PEDAGÓGICOS EN EDUCACIÓN FÍSICA Y LA EVALUACIÓN FORMATIVA
}

The hybridisation of pedagogical models in physical education and the formative assessment

A hibridação de modelos pedagógicos em educação física e a avaliação formativa

\section{Ángel Pérez Pueyo (1)}

\section{David Hortigüela Alcalá (2)}

Israel Herrán Álvarez (3)

David Vega Cobo (4)

\section{Carlos Heras Bernardino (5) \\ Jorge Garrote García (6) \\ Mario Sobejano Carrocera (7)}

\section{Alejandra Hernando Garijo (8)}

(1) Universidad de León, +34 619867767, angel.perez.pueyo@unileon.es

(2) Facultad de Educación. Universidad de Burgos, +34 9472595 17, dhortiguela@ubu.es

(3) IES Doctor Sancho de Matienzo, +34 665016492, israeleducacionfisica@gmail.com

(4) CP Menéndez Pidal, Bembibre (León), +34 658777538, davidvegacobo@hotmail.com

(5) IES Prado de Santo Domingo, Alcorcón (Madrid), +34 669796038, carlos.herasbernardino@educa.madrid.org

(6) IES Lancia (Leon), +34 665016492, jorge.garrote@hotmail.com

(7) IES Nueve Valles, Torrelavega, (Cantabria), +34 620920886, mario.sobejano@gmail.com

(8) Facultad de Educación. Universidad de Burgos, +34 649690858, ahgarijo@ubu.es 


\title{
Resumen
}

La experiencia presenta la hibridación de modelos pedagógicos como el Estilo Actitudinal de educación física, el Aprendizaje cooperativo de carácter general y técnicas relacionadas con el e-learning como el Flipped-classroom, vinculadas todas ellas a las denominadas metodologías activas. Ésta es llevada a cabo con el aprendizaje de cabuyería y franqueamiento de obstáculos en relación al medio natural, en contextos tan diferentes pero íntimamente relacionados como los centros de primaria, los de secundaria y los de formación de futuros docentes de educación física. La actividad, con un alto carácter cooperativo, muestra la realidad de la interdependencia positiva entre los miembros del grupo. Además, el proceso de evaluación formativa llevado a cabo, a través de una escala de valoración y la coevaluación intragrupal, permite comprobar al alumnado el potencial educativo de los procesos de aprendizaje compartido utilizando de un instrumento de evaluación objetivo entregado al inicio de la unidad.

Palabras clave: Hibridación; modelos pedagógicos; cabuyería; evaluación formativa; escala de valoración

\begin{abstract}
This experience shows the hybridisation of different pedagogical models such as the Attitudinal Style specific from physical education (P.E.), the Cooperative Learning from a general perspective and the techniques related with e-learning such as FlippedClassroom, which are all related to the commonly called active methodologies. Our proposal is accomplished through the learning of knot tying and surpassing obstacles in activities related with the natural environment. Such proposal has been implemented in different but related contexts, such as primary schools, high schools and training schools for future P.E. teachers. This activity, which is highly cooperative, shows how real the positive interdependence among the members of the group can be. Moreover, the formative assessment process developed, through an assessment scale and the peerassessment among the members of the group, allows the students to check the learning possibilities of shared learning processes using an objective assessment instrument handed in at the beginning of the lesson plan.
\end{abstract}

Keywords: Hybridisation; pedagogical models; knot tying; formative assessment; assessment scale 


\section{Resumo}

A experiência mostra a hibridização de modelos pedagógicos, tais como a educação do Comportamento Estilo física, aprendizagem geral cooperativa e relacionado a como técnicas de sala de aula Flipped, todos eles ligados aos chamados metodologias ativas de e-learning. Isso é feito com a aprendizagem cabuyería e obstáculo em relação ao ambiente natural, tão diferentes, mas estreitamente ligados contextos como escolas primárias, secundárias e na formação dos futuros professores de educação física. Atividade, com alta de caráter cooperativo, mostra a realidade da interdependência positiva entre os membros do grupo. Além disso, o processo de avaliação formativa realizada através de uma avaliação por pares escala de classificação e intragrupos, permite verificar que os alunos o potencial educativo do processo de aprendizagem compartilhada usando um instrumento de avaliação objetiva entregue no início da unidade.

Palavras-chave: Hibridação; modelos pedagógicos; cabuyería; avaliação formativa; escala de classificação

\section{Introducción}

Uno de los aspectos importantes en la formación del profesorado en el Espacio Europeo de Educación Superior (EEES) se basa en que éste experimente metodologías innovadoras que le doten de los recursos para ser autónomos (Zabala, 2006) consiguiendo una mayor vinculación entre la formación académica y la cualificación profesional (Romero, 2008). En este sentido, Barragán (2005) comenta la importancia de innovar metodologías, recursos y productos que permitan al universitario y, en este caso futuro docente, alcanzar las competencias establecidos por el EEES. En este sentido, el conocimiento y la hibridación de modelos pedagógicos (Fernández-Río, Calderón, Hortigüela, Pérez-Pueyo \& Aznar, 2016) se antoja fundamental en la actualidad.

En este caso, el Estilo Actitudinal (2010) y el Aprendizaje Cooperativo (Johnson \& Johnson, 1999; Velázquez, 2004) son algunos de los modelos pedagógicos que ponen el acento en el aprendizaje del alumno haciendo que los futuros docentes necesiten experimentarlos para poder ponerlos en práctica con garantías de éxito (Pérez-Pueyo, 
Herrán \& Hortigüela, 2016). Además, su combinación con otro modelo pedagógico, aunque en este caso no específico de la Educación Física como es el denominado Flipped Classroom (Bergmann \& Sams, 2012; 2014; Tourón, Santiago, \& Díez, 2014) ofrece la posibilidad de convertir los videotutoriales en generadores iniciales del conocimiento necesario para que se produzca el aprendizaje. Esta hibridación de modelos nos acerca hacia las propuestas innovadoras en las que el alumnado experimenta de manera simultánea metodologías contrastadas.

\section{Contextualización}

La experiencia se lleva a cabo en la asignatura de Aprendizaje de las materias correspondientes del Módulo de Educación Física de Máster Universitario de Formación de Profesorado en Educación Secundaria Obligatoria y Bachillerato, Formación Profesional y Enseñanzas de la Universidad de León, en la Facultad de Educación de Burgos, así como en el IES Doctor Sancho de Matienzo de Villasana de Mena (Burgos), IES Prado de Santo Domingo, en Alcorcón (Madrid), en el IES Lancia (Leon), IES Nueve Valles Torrelavega, (Cantabria) y en el CP Menéndez Pidal de Bembibre (León). En todos los casos, el trabajo se lleva a cabo siguiendo las mismas pautas de actuación y llevando a cabo el mismo proceso de experimentación y aprendizaje.

\section{Diseño y desarrollo}

Tanto en el Máster y en la Facultad de Educación como en los cursos de $4^{\circ}$ de la ESO y de $6^{\circ}$ de Primaria, comenzamos proporcionando a los alumnos o una ficha o un enlace de video donde puede ver y aprender cómo se realizan los nudos. Y a partir de ese momento, se organizan las sesiones en los siguientes pasos:

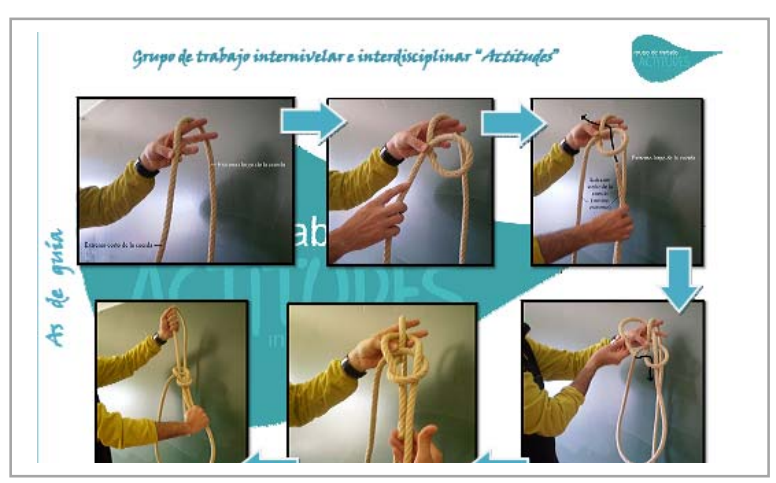

Figura 1.

Ficha de As de Guía 
$1^{\circ}$.- Cada grupo, de cuatro personas, debe repartirse los nudos (uno por miembro del grupo: As de guía, Ballestrinque, Siete y medio, y Ocho doble) (Pérez-Pueyo \& Casado, 2014) con el objetivo de que cada uno se haga experto en el que le haya correspondido.

$2^{\circ}$.- En casa y utilizando el videotutorial del nudo subido al canal del youtube del “Grupo Actitudes”, cada miembro debía aprender el nudo que le había correspondido convirtiéndose en experto para enseñarlo posteriormente a los compañeros de su grupo.

$3^{\circ}$.- El primer día de clase, a través de la técnica cooperativa denominada "El puzzle de Aronson” y antes de juntarse con los compañeros de su grupo, todos los expertos de cada nudo se juntarán en un "grupo de expertos” (uno de cada grupo) con la intención de asegurarse de que todos los expertos se saben perfectamente el nudo y cómo se realiza y explica. En caso de que alguno no lo domine, el grupo de expertos se asegurará que todos lo acaban aprendiendo perfectamente antes de ir a su grupo de referencia a explicarlo.

$4^{\circ}$.- En su grupo de referencia, cada experto en un nudo se asegura de que todos los compañeros aprenden el suyo y viceversa; consiguiendo que al final los cuatro miembros se sepan los cuatro nudos a aprender.

$5^{\circ}$.- Posteriormente, se le propone al grupo un reto: el tensado de una cuerda entre dos árboles lo más firme posible (figura 2) (Pérez-Pueyo \& Casado, 2014). La intención es que su tensión permita prácticamente andar por encima de ella sin que se combe.

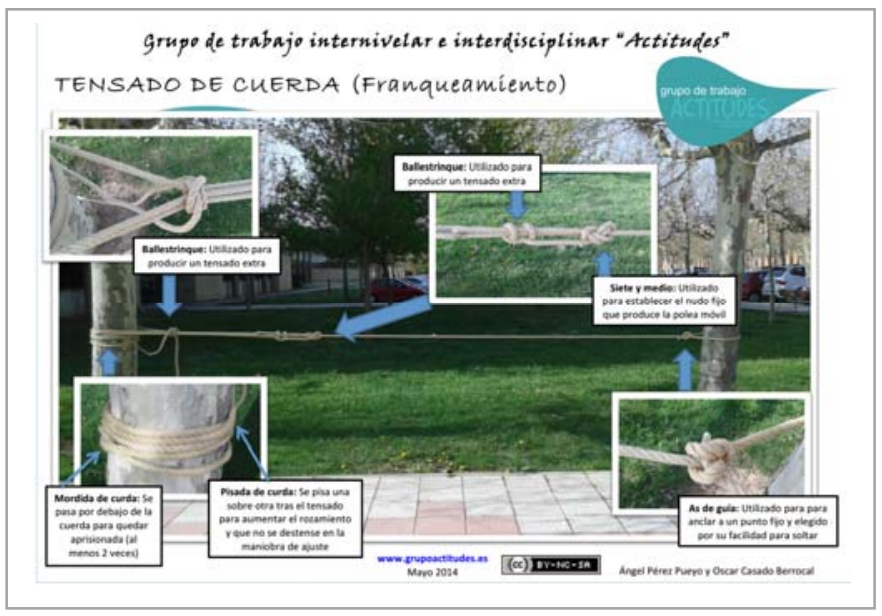

Figura 2.

Reto: Tensado del puente

$6^{\circ}$.- Cuando todos han aprendido a realizar el tensado, se les proporciona una escala de valoración (Pérez-Pueyo \& Casado, 2016) (figura 3) que les permite preparar la 
realización de la actividad de evaluación que, además, deberá ser grabada para constatar la evidencia del aprendizaje y la posterior valoración de la misma con el mismo instrumento

\section{Evaluación y resultados}

Este $6^{\circ}$ paso conlleva la valoración del aprendizaje conseguido. En este sentido, independientemente del grupo (universitario o no universitario), antes del tensado los alumnos tuvieron la oportunidad de practicar llevando a cabo procesos de autoevaluación y coevaluación desde un enfoque formativo.

\begin{tabular}{|l|c|c|}
\hline \multicolumn{3}{|l|}{} \\
\hline $\begin{array}{l}\text { Escala de valoración para el tensado de un puente (franquesmiento de } \\
\text { obstulos) }\end{array}$ & Evaluación & Calif \\
\hline Realización del As de guía & Muy bien & 10 \\
\hline $\begin{array}{l}\text { Realizar con rapidez y soltura el nudo en diferentes } \\
\text { posiciones }\end{array}$ & Bien & 8 \\
\hline Realiza el nudo correctamente & Regular & 5 \\
\hline Titubea a realizar el nudo pero lo consigue hacer & 0 \\
\hline No lo consigue realizar & Muy bien & 10 \\
\hline Realización del Ballestrinque & Bien & 8 \\
\hline $\begin{array}{l}\text { Realizar con rapidez y soltura el nudo en diferentes } \\
\text { posiciones }\end{array}$ & Regular & 5 \\
\hline Realiza el nudo correctamente & Mal & 0 \\
\hline Titubea a realizar el nudo pero lo consigue hacer & Muy bien & 10 \\
\hline No lo consigue realizar & Bien & 6 \\
\hline Realización del Siete y medio & Mal & 0 \\
\hline Realiza el nudo correctamente & & $\mathbf{7 0}$ \\
\hline Titubea a realizar el nudo pero lo consigue hacer & Excelente & 70 \\
\hline No lo consigue realizar & Pobre & 20 \\
\hline Realización del tensado del puente & Mal & 0 \\
\hline $\begin{array}{l}\text { Coloca los nudos adecuados en cada zona del tensado, } \\
\text { los ejecuta y sitúa correctamente y tensa la cuerda } \\
\text { realizando el ajuste y cierre final perfectamente }\end{array}$ & 55 \\
\hline $\begin{array}{l}\text { Coloca los nudos adecuados en cada zona del tensado, } \\
\text { los ejecuta con algún titubeo o no los sitúa exactamente } \\
\text { en el lugar correcto y tensa la cuerda realizando el ajuste } \\
\text { y cierre final }\end{array}$ & Muy bien & 55 \\
\hline $\begin{array}{l}\text { Coloca los nudos adecuados en cada zona del tensado, } \\
\text { los ejecuta con algún titubeo y no los sitúa exactamente } \\
\text { en el lugar correcto, y tensa la cuerda aunque el ajuste y } \\
\text { cierre final es mejorable }\end{array}$ & Bien & 30 \\
\hline $\begin{array}{l}\text { Duda en la colocación de los nudos adecuados en cada } \\
\text { zona del tensado, los ejecuta con algún titubeo y no los } \\
\text { sitúa en el lugar correcto; y, además, tensa la cuerda } \\
\text { aunque no ajustándola y el cierre final es mejorable o se } \\
\text { olvida }\end{array}$ & Regular \\
\hline $\begin{array}{l}\text { Aunque parece que está tensado, hay nudos mal } \\
\text { realizados }\end{array}$ & & \\
\hline No consigue que la cuerda se tense o montar el puente & & \\
\hline & & \\
\hline
\end{tabular}

Figura 3.

Escala de valoración de los nudos y el tensado en $4^{\circ}$ de la ESO

El reto del tensado pretende generar la aplicación de los conocimientos adquirido; sin embargo, la utilización de la escala de valoración tiene por intención la comprobación y asentamiento del aprendizaje. Y lo más interesante es que los procesos de coevaluación intragrupal, conducentes a la grabación de la evidencia de aprendizaje tanto individual como grupal, consiguen que todos y cada uno de los alumnos demuestren el aprendizaje adquirido. 
En cada uno de los contextos de aplicación, con independencia de la edad del alumnado, todos los alumnos demostraron haber aprendido los nudos y fueron capaces de tensar la cuerda entre dos árboles aplicando los nudos aprendidos cooperativamente

\section{Conclusiones}

La experiencia internivelar ha demostrado que la metodología, relacionada con la evaluación formativa, es capaz de lograr el resultado esperado. En este sentido, y como es habitual en el Estilo Actitudinal, el logro fue de todos; sin excepción y en cada una de las etapas y grupos llevados a cabo.

\section{Referencias}

Barragán, R. (2005). El Portafolio, metodología de evaluación y aprendizaje de cara al nuevo Espacio Europeo de Educación Superior. Una experiencia práctica en la Universidad de Sevilla. Revista Latinoamericana de Tecnología Educativa, 4(1), 121-139. Recuperado de http://www.unex.es/didactica/RELATEC/ sumario_4_1.htm.

Johnson, D.W., \& Johnson, R.T. (1999). Aprender juntos y solos. Aprendizaje cooperativo, competitivo e individualista. Buenos Aires: Aique.

Pérez-Pueyo, A., \& Casado, O. M. (2014). Fichas de cabuyería [documento de descarga]. $\quad$ Recuperado de https://drive.google.com/drive/u/0/folders/0B6zM2sfGO-

\section{LsVTZOTWhCdmNMaFE}

Pérez-Pueyo, A., \& Casado, O. M. (2016). Escala de valoración para nudos en cabuyería [documento de descarga]. Recuperado de https://drive.google.com/file/d/0B7xEdPlO4dj8SWMzcnE3SFAwNEE/view

Pérez-Pueyo, A., Herrán, I., \& Hortigüela, D. (2016). Tres nudos: el franqueamiento de obstáculos desde el Estilo Actitudinal. D. Pérez-Brunicardi, J. Frutos, P. Caballero, A. Baena-Extrenera \& A. Miguel-Aguado. Actas de las jornadas sobre actividades en la naturaleza. Todos juntos, coun un mismo rumbo (pp. 99-108). Segogia: Red Estatal de Educación Física en la Naturaleza (REEFN). 
Romero, C. (2008). El portafolios y el trabajo de grupo: una experiencia del crédito ECTS en la formación del magisterio especialista en Educación Física. REIFOP, 11(2), 1-12 Recuperado de http://www.aufop.com/aufop/home/

Rué, J. (2007). Enseñar en la Universidad. El EEES como reto para la Educación Superior. Madrid: Narcea.

Zabalza, M. A. (2006). La convergencia como oportunidad para mejorar la docencia universitaria. Revista Interuniversitaria de Formación del Profesorado 20(3), 3769. 